\section{HPV als Auslöser von Spinaliomen?}

Bei immunsupprimierten Patienten mit nichtmelanotischem Hautkarzinom sind häufig Infektionen mit humanen Papillomviren (HPV) der Betagruppe nachweisbar. Spielen die Viren auch bei anderen Patienten eine Rolle bei der Karzinogenese?

D aten zum Vorkommen von HPV bei immunkompetenten Patienten mit nichtmelanotischem Hautkrebs (NMHK) sind rar und stammen vor allem aus epidemiologischen Studien, die keine Aussage über die zeitliche Abfolge von Infektion und Krebsentstehung zulassen. Deswegen haben Ärzte aus Rom das Auftreten eines NMHK in Abhängigkeit vom HPV-Status prospektiv untersucht.

Weil dafür bei gesunden Studienteilnehmern eine sehr große Zahl von Personenjahren erforderlich wäre, wählten sie Patienten, die wegen eines NMHK in der Anamnese ein erhöhtes Risiko für eine erneute Erkrankung hatten. 107 Patienten einer früheren Fall-Kontroll-Studie wurden nach Ablauf von fünf Jahren erneut untersucht. Von ihnen hatten 58 ein Plattenepithelkarzinom und 49 ein Basalzellkarzinom gehabt. Für alle Patienten lagen serologische Daten zur Infektion mit 28 verschiedenen HPV-Typen vor.

Die Folgeuntersuchung ergab, dass Seropositivität für eine Reihe von betaund gamma-HPV (aber nicht für andere Virustypen) mit einem erhöhten Risiko für ein erneutes Plattenepithelkarzinom einherging. Das Risiko war allerdings nur bei Patienten mit einem früheren Plattenepithelkarzinom signifikant. Wenn diese Patienten Antikörper etwa gegen HPV-24 aufwiesen, hatten sie ein vierfach erhöhtes Risiko für ein weiteres Plattenepithelkarzinom. Keinerlei Zusammenhang zeigte sich dagegen zwischen dem serologischen HPV-Status und der Entwicklung eines Basalzellkarzinoms.

Fazit: Patienten mit einem Plattenepithelkarzinom der Haut in der Vorgeschichte entwickeln häufiger einen erneuten Tumor dieser Arzt, wenn sie seropositiv für beta- oder gamma-HP-Viren sind. Dieses Ergebnis stützt Beobachtungen aus FallKontroll-Studien zu einem möglichen kausalen Zusammenhang zwischen HPV und NMHK. Dr. Beate Schumacher

Paradisi A et al. Seropositivity for human papillomavirus and incidence of subsequent squameous cell and basal cell carcinomas of the skin in patients wiht a previous nonmelanoma skin cancer. Br J Dermatol 2011;165: 782-91

\section{Retikuläre erythematöse Muzinose: Chloroquine als erfolgreiche Therapie}

Wie die retikuläre erythematöse Muzinose entsteht, ist nicht bekannt. Was gegen die Erkrankung hilft, hingegen schon: Antimalariamittel wie Chloroquin und Hydroxychloroquin. Bochumer Dermatologen haben die Wirksamkeitder Substanzen überprüft.

$\mathrm{D}$ ie retikuläre erythematöse Muzinose (REM), auch Rundzellerythematose genannt, befällt vor allem Brust und Rücken, bei Frauen doppelt so häufig wie bei Männern. Das Lehrbuch von Altmeyer nennt als Symptome: netzförmige bis flächenhafte, scharf begrenzte, unregelmäßig

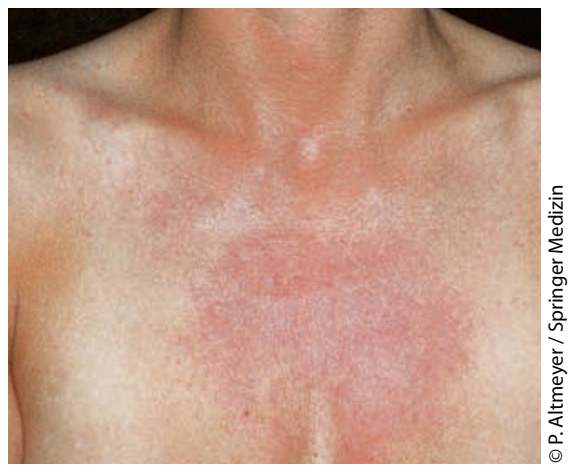

Retikuläre erythematöse Muzinose konfigurierte, hellrote, selten bräunliche, ggf. leicht urtikarielle Erytheme und $\mathrm{Pa}$ peln. Gelegentlich besteht Juckreiz. Bei etwa 30\% der Patienten exazerbiert der Hautbefund nach Sonnenexposition.

Die klinischen Daten von elf dieser Patienten, zehn davon Frauen, hat ein Team um Alexander Kreuter von der RuhrUniversität nun retrospektiv analysiert. Zehn der Probanden waren Raucher, sechs hatten eine begleitende Autoimmunerkrankung (Hashimoto-Thyreoiditis, Diabetes mellitus, Psoriasis-Arthritis, rheumatoide Arthritis). Alle Patienten hatten gegen ihre REM eines der Antimalariamittel Chloroquin oder Hydroxychloroquin erhalten.

Mangels eines eigenen klinischen Scores für REM bedienten sich Kreuter und Kollegen des „Cutaneous Lupus Erythematosus Disease Area and Severity
Index" (CLASI), um den Therapieerfolg zu beurteilen. Tatsächlich sank der Score nach dreimonatiger Antimalaria-Behandlung von median 4 auf 0 und veränderte sich auch bis zum zwölften Beobachtungsmonat nicht.

Fazit: Antimalariamittel verbessern das Hautbild bei REM signifikant oder lassen die Läsionen sogar völlig verschwinden. Chloroquin und Hydroxychloroquin sollten deshalb als Erstlinientherapie dieser seltenen Erkrankung betrachtet werden.

Dr. Robert Bublak

Kreuter A et al. Clinical Features and Efficacy of Antimalarial Treatment for Reticular Erythematous Mucinosis. Arch Dermatol 2011; 147: $710-5$ 